\title{
Which mouthrinses are the most effective at plaque prevention?
}

\author{
An in vitro evaluation of the availability of cetylpyridinium chloride and chlorhexidine in some commercially \\ available mouthrinse products. S. Sheen and M. Addy Br Dent J 2003; 194: 207-210
}

\section{Objectives}

To assess the comparative activity of mouthrinses containing cetylpyridinium chloride (CPC) and chlorhexidine using the propensity to cause extrinsic staining in vitro as the outcome variable.

\section{Methods}

Saliva-coated clear acrylic specimens were exposed to cyclical staining regimens of either CPC or chlorhexidine followed by tea. Water and $0.2 \%$ chlorhexidine were used as negative and positive controls respectively. Staining cycles were repeated until an optical density of $>2$ was reached by one of the products.

\section{Results}

For CPC there was a highly significant difference in staining between the products. Two CPC products performed numerically little better than water. For the chlorhexidine products the $0.2 \%$ formulation produced the most staining although little more than the UK version of the $0.1 \%$ rinse. The French $0.1 \%$ rinse produced by the same manufacturer as the UK formulation showed markedly reduced staining potential although significantly greater than water.

\section{Conclusion}

This study, supported by previous in vitro and in vivo studies, indicates discrepancies in the availability of CPC and chlorhexidine in some mouthrinse products. Importantly, this may have an effect on the potential of some rinses to provide the expected plaque inhibitory activity.

\section{IN BRIEF}

- This study hightlights the fact that some commercially available mouthrinses may not achieve the plaque inhibitory activity that they are expected to have.

- Ingredients contained within some cationic antiseptic mouthrinse solutions may inactivate the plaque inhibitory agent.

- The study confirms the varying ability of different mouthrinses to form stains in the laboratory and this has been found in many previous studies to be indicative of clinical staining and plaque inhibitory efficacy.

\section{COMMENT}

This in vitro study reports discrepancies in the availability of cetylpyridinium chloride (CPC) and chlorhexidine in some commercially available mouthrinses based on extrinsic staining. Mouthrinses containing plaque inhibitory ingredients are useful adjuncts to mechanical supraginigival plaque control and may be especially important when this is difficult to perform. Many formulations of mouthrinse contain the CPC as the active ingredient although chlorhexidine remains the acknowledged 'gold standard'. However, the same active ingredient in different formulations does not mean one product will inhibit plaque as effectively as another. Other ingredients may modify plaque inhibition, particularly the addition of detergents to mouthrinses.

Twelve CPC and two chlorhexidine $0.1 \%$ containing mouthrinses were investigated. A $0.2 \%$ chlorhexidine rinse and distilled water acted as positive and negative standards. Briefly, perspex discs were treated with human saliva, the mouthrinse solution being tested, and finally exposed to tea. The dried perspex specimens were read on a spectrometer and the whole process repeated until the average optical density reading of six blocks of one or more groups at that cycle reached a value of $>2.0$. The data for one product (Dentyl Ph) was discarded due to invalid optical readings.

For CPC containing mouthrinses a highly significant difference in staining was found between the products tested and two were similar to water in this respect. The concentration of CPC was stipulated in two formulations as $0.05 \%$, which is the most common concentration used commercially. For chlorhexidine containing mouthrinses, the amount of staining was similar for the UK version of a $0.1 \%$ rinse to the $0.2 \%$ formulation, whereas the French $0.1 \%$ rinse tested showed markedly reduced staining in comparison.

These findings based on extrinsic staining indicate discrepancies in the availability of CPC and chlorhexidine between commercially available mouthrinses. These findings are of interest and it is important for clinicians to be aware that products with the same active ingredient may not have the same degree of plaque inhibition. A previous study has used in vitro extrinsic staining to position clinical efficacy in mean terms for chlorhexidine containing mouthrinses in order, however the authors wisely caution that clinical trials are required to support the inferences made in the present study.

Andrew Rawlinson, Senior Lecturer in Restorative Dentistry, School of Clinical Dentistry, University of Sheffield. 